\title{
Explicit Dehn filling and Heegaard splittings
}

\author{
David Futer and Jessica S. Purcell
}

\begin{abstract}
We prove an explicit, quantitative criterion that ensures the Heegaard surfaces in Dehn fillings behave "as expected." Given a cusped hyperbolic 3-manifold $X$, and a Dehn filling whose meridian and longitude curves are longer than $2 \pi(2 g-1)$, we show that every genus $g$ Heegaard splitting of the filled manifold is isotopic to a splitting of the original manifold $X$. The analogous statement holds for fillings of multiple boundary tori. This gives an effective version of a theorem of Moriah-Rubinstein and Rieck-Sedgwick.
\end{abstract}

\section{Introduction}

In 1997, Moriah and Rubinstein investigated the relationship between Heegaard splittings of a cusped hyperbolic 3-manifold and the Heegaard splittings of its Dehn fillings [16]. They showed that if one imposes a bound on the genus of the surfaces and excludes finitely many Dehn filling slopes, then every irreducible Heegaard surface in the filled manifold is isotopic to one of a finite collection of surfaces in the original manifold. In 2001, Rieck and Sedgwick used topological ideas to show that any Dehn filling slope that results in a smaller genus Heegaard surface must lie on one of a finite number of so-called "bad" slopes and "destabilization lines" in Dehn surgery space [20]. Rieck showed that the number of bad slopes is bounded by a quadratic function of the genus [18], while Rieck and Sedgwick showed that this number is finite in general, independent of genus [19].

Due to this previous work, we know that if we exclude a finite number of Dehn filling slopes, and a finite number of destabilization lines in the Dehn surgery space, any bounded-genus Heegaard surface in a Dehn filling will be a Heegaard surface in the original manifold. However, there has not been an effective characterization of which slopes and destabilization lines must be excluded. As a consequence, it has been difficult to use these results to prove explicit bounds, such as those needed in a recent paper of the authors with Cooper [6].

In this paper, we make these constraints explicit. In particular, we show the following. 
Theorem 1.1. Let $X$ be a cusped, orientable hyperbolic 3-manifold. Choose disjoint horospherical neighborhoods $C_{1}, \ldots, C_{k}$ about some subset of the cusps, and let $s_{i}$ be a Dehn filling slope on each torus $\partial C_{i}$. Let $\Sigma$ be a Heegaard surface of genus $g \geq 1$ for the Dehn filled manifold $M=X\left(s_{1}, \ldots, s_{k}\right)$. Then we have the following.

1) If the length $\ell\left(s_{i}\right)$ satisfies $\ell\left(s_{i}\right)>2 \pi(2 g-1)$ for every $i$, then each core curve $\gamma_{i}$ for the filling solid torus is isotopic into $\Sigma$ (although these cores may not be simultaneously isotopic into $\Sigma$ ).

2) If, in addition, the shortest longitude $\lambda_{i}$ for each $s_{i}$ satisfies $\ell\left(\lambda_{i}\right)>$ $6(2 g-3)$, the surface $\Sigma$ can be isotoped into $M \backslash\left(\gamma_{1} \cup \ldots \cup \gamma_{k}\right) \cong X$, and forms a Heegaard surface for $X$.

A longitude for $s_{i}$ in item (2) is defined to be a slope $\lambda_{i}$ on $\partial C_{i}$ that intersects $s_{i}$ once. The shortest longitude is a longitude whose length is smallest among all longitudes for $s_{i}$. Here the lengths of $s_{i}$ and $\lambda_{i}$ are the lengths of geodesic representatives on the horospherical torus $\partial C_{i}$, in the metric induced by the hyperbolic metric on $X$.

The slopes on $\partial C_{i}$ that fail conclusion (1) of the theorem, i.e., those slopes whose core of the Dehn filling solid torus is not isotopic into $\Sigma$, are exactly the "bad" slopes studied by Rieck and Sedgwick [18-20]. Thus part (1) of Theorem 1.1 gives an explicit finite list of candidates for bad slopes. Similarly, for each slope $\lambda_{i}$ of length less than $6(2 g-3)$, the primitive integer coordinates of the meridians that intersect $\lambda_{i}$ once lie on a single line in $\mathbb{R}^{2}$; this line is exactly the "destabilization line" corresponding to $\lambda_{i}$. Thus part (2) of Theorem 1.1 gives an explicit finite list of candidates for destabilization lines.

We note that because $g \geq 1$, the hypotheses of the theorem always require that the Dehn filling slopes satisfy $\ell\left(s_{i}\right)>2 \pi$. As a result, the $2 \pi$-Theorem of Gromov and Thurston [2] implies that the manifold $M=X$ $\left(s_{1}, \ldots, s_{k}\right)$ has a negatively curved metric. (Thus, in fact, the case $g=1$ is vacuous.)

Our main tool in proving Theorem 1.1 is geometry: we obtain our conclusions from area considerations in the negatively curved metric on $M$. This geometric viewpoint follows the lead of Moriah and Rubinstein's paper [16]. We also follow their lead in using a theorem of Pitts and Rubinstein [17, 21] that relates Heegaard surfaces to minimal surfaces in $M$ (see Lemma 3.8 below). The Pitts-Rubinstein result has been used in a number of applications (e.g., $[3,12,14,16]$ ), but unfortunately a complete proof of this result does not appear in the literature. De Lellis and Pellandini have proved an 
important step, namely that the "minimax method" produces a minimal surface of the appropriate genus [7]. In addition, a survey paper by Souto [28] contains a discussion of the status of the proof, including what remains to be done after the work of De Lellis and Pellandini. Souto claims in [28] to have worked out the remainder of the proof, although his argument has not yet appeared. In this work, we shall assume the Pitts-Rubinstein result, in anticipation of a full proof.

In addition to methods used by Moriah and Rubinstein, our argument takes advantage of several other tools, some of which were unavailable in 1997.

First, we will use an effective version of the $2 \pi$-Theorem, due to the authors and Kalfagianni [9, Theorem 2.1], to get explicit estimates on curvature and area in $M$. This result, described in Section 2, will give us much more effective control over surfaces.

Second, we will use the notion of generalized Heegaard splittings, developed by Scharlemann and Thompson [25], to reduce the crux of the argument to the case where $\Sigma$ is strongly irreducible. We review the relevant ideas in Section 3 .

Third, our proof relies on the argument in a recent paper by Breslin [3]. In fact, we obtain a generalization of his theorem, which is likely to be of independent interest. To simplify the statement of our generalization, we use the following definition.

Definition 1.2. Let $V$ be a solid torus, with a prescribed Riemannian metric. We say the metric on $V$ is submersible if, after lifting the metric to the universal cover $\widetilde{V} \cong D \times \mathbb{R}$, there is a Riemannian submersion from $\widetilde{V}$ to its cross-sectional disc $D$. Recall that a smooth map $f: \widetilde{V} \rightarrow D$ is called a Riemannian submersion if its differential $d f: T_{p} \widetilde{V} \rightarrow T_{f(p)} D$ is an orthogonal projection at each point.

We note that if $M$ has a hyperbolic metric and $V \subset M$ is a fixed-radius tube about a closed geodesic, then the metric on $V$ is submersible. In addition, we will see in Section 2 that the negatively curved solid tori constructed using the $2 \pi$-Theorem are also submersible. Thus the hypothesis of submersibility is relatively mild.

Theorem 1.3. Let $M$ be an orientable Riemannian 3-manifold whose noncompact ends (if any) are isometric to horospherical cusp neighborhoods, and whose boundary (if any) consists of minimal surfaces. Suppose the sectional curvatures of $M$ are bounded above by $\kappa_{\max }<0$. Let $\Sigma$ be a Heegaard surface 
for $M$. Let $V$ be a solid torus in $M$, such that the metric on $V$ is submersible and its cross-sectional disc $D$ satisfies

$$
\operatorname{area}(D)>2 \pi \chi(\Sigma) / \kappa_{\max }
$$

Then the core curve $\gamma$ of $V$ is isotopic into $\Sigma$. Here $\chi(\Sigma)$ denotes Euler characteristic.

Theorem 1.3 generalizes Breslin's theorem in several ways. First, it does not require $\Sigma$ to be strongly irreducible. Second, it allows $M$ to have cusps and/or boundary. Third, it allows the metric on $M$ to have variable curvature. Finally, the explicit hypothesis of Equation (1.1) may be easier to check in applications than Breslin's hypothesis on the length of $\gamma$. These improvements are obtained by modifications of Breslin's original argument; we describe them in Section 4.

With all of this work in hand, part (1) of Theorem 1.1 will follow immediately by applying Theorem 1.3 to the negatively curved metric described in Theorem 2.1. Part (2) of Theorem 1.1 will follow by another geometric argument, which is given in Section 5.

\section{A negatively curved metric on the filled manifold}

In this section, we begin with a cusped hyperbolic manifold $X$, and recall an explicit construction of a negatively curved metric on a Dehn filling $M=X\left(s_{1}, \ldots, s_{k}\right)$. Following results of the the authors and Kalfagianni in [9, Section 2], we will obtain explicit estimates on curvature and areas in the negatively curved metric on $M$. These estimates are designed to plug into Equation (1.1), which will give us control over Heegaard surfaces in $M$.

Theorem 2.1. Let $X$ be a complete, finite-volume hyperbolic manifold with cusps. Suppose $C_{1}, \ldots, C_{k}$ are disjoint horoball neighborhoods of some (possibly all) of the cusps. Let $s_{1}, \ldots, s_{k}$ be slopes on $\partial C_{1}, \ldots, \partial C_{k}$, each with length greater than $2 \pi$. Denote the minimal slope length by $\ell_{\min }$.

Then, for every $\zeta \in(0,1)$, the Dehn filled manifold $M=X\left(s_{1}, \ldots, s_{k}\right)$ admits a Riemannian metric, in which the cusp $C_{i}$ is replaced by a negatively curved solid torus $V_{i}$. This metric has the following properties:

1) The metric on $M \backslash \bigcup_{i} V_{i}$ agrees with the hyperbolic metric on $X \backslash \bigcup_{i} C_{i}$.

2) The sectional curvatures of $M$ are bounded above by $\zeta\left(\left(\frac{2 \pi}{\ell_{\min }}\right)^{2}-1\right)<0$. 
3) The metric on each $V_{i}$ is submersible, as in Definition 1.2.

4) The cross-sectional disc of $V_{i}$ has area at least $\zeta\left(\frac{\ell_{\min }{ }^{2}}{\ell_{\min }+2 \pi}\right)$.

Proof. To construct a Riemannian metric on $M$ satisfying (1), it suffices to construct a negatively curved metric on each solid torus $V_{i}$, such that in a collar neighborhood of $\partial V_{i}$, it agrees with the hyperbolic metric in a collar neighborhood of $\partial C_{i}$. This is precisely what is done in [9, Theorem 2.1]. Thus the cusp neighborhoods $C_{i}$ can be replaced by solid tori $V_{i}$, obtaining (1). In addition, we showed in [9, Lemma 2.3 and Theorem 2.5] that the curvatures of the resulting metric on $M$ are bounded as claimed in (2).

For the rest of the argument, we focus on one solid torus $V=V_{i}$, and drop the subscripts for convenience. We describe the metric on $V$ that was constructed in $\left[9\right.$, Theorem 2.1]. Let $t_{\text {lim }}=1-\left(2 \pi / \ell\left(s_{i}\right)\right)^{2}$, and choose a parameter $t \in\left(0, t_{\mathrm{lim}}\right)$. For fixed $t$, the metric on $V$ can be described in cylindrical coordinates by the equation

$$
d s^{2}=d r^{2}+\left(f_{t}(r)\right)^{2} d \mu^{2}+\left(g_{t}(r)\right)^{2} d \lambda^{2},
$$

Here, $f_{t}$ and $g_{t}$ are functions obtained by solving a certain ODE. The coordinate $0 \leq \mu \leq 1$ is measured around each meridional circle, while $0 \leq \lambda \leq 1$ is measured perpendicular to $\mu$ in the longitudinal direction. Furthermore, $r_{0}(t) \leq r \leq 0$ is radial distance, where $r=0$ on the boundary torus $\partial V$ and $r_{0}(t)<0$ is the unique root of the function $f_{t}(r)$. The radial value $r=r_{0}(t)$ corresponds to the core of the solid torus $V$.

Observe that the expression for the metric in Equation (2.1) is already diagonalized, with the three coordinate vectors mutually orthogonal. Furthermore, if we lift the metric to the universal cover $\widetilde{V}$, the $\mu$ and $\lambda$ coordinates are globally defined. Thus the projection from $\widetilde{V} \cong D \times \mathbb{R}$ to the cross-sectional disc $D$, defined by $(r, \mu, \lambda) \mapsto(r, \mu)$, is a Riemannian submersion, as claimed in (3).

To prove the theorem, it remains to compute the area of a meridian disc $D$. Since the coordinate $\lambda$ is constant over a meridian disc, the area for fixed $t$ will be given by

$$
\int_{0}^{1} \int_{r_{0}(t)}^{0} f_{t}(r) d r d \mu=\int_{r_{0}(t)}^{0} f_{t}(r) d r
$$


By [9, Theorem 5.4 and Equation (4)], we know that as $t \rightarrow t_{\text {lim }}$, the functions $f_{t}$ converge uniformly to

$$
f_{t_{\mathrm{lim}}}(r)=\frac{\ell\left(s_{i}\right) \sqrt{1-t_{\mathrm{lim}}}}{\sqrt{t_{\mathrm{lim}}}} \sinh \left(\sqrt{t_{\mathrm{lim}}}\left(r-r_{0}\right)\right),
$$

where

$$
r_{0}=-\tanh ^{-1}\left(\sqrt{t_{\text {lim }}}\right) / \sqrt{t_{\text {lim }}} .
$$

Thus, as $t \rightarrow t_{\text {lim }}$, the area of the meridian disc limits to

$$
\begin{aligned}
\lim _{t \rightarrow t_{\lim }} \int_{r_{0}(t)}^{0} f_{t}(r) d r & =\int_{r_{0}}^{0} f_{t_{\lim }}(r) d r \\
& =\int_{r_{0}}^{0} \frac{\ell\left(s_{i}\right) \sqrt{1-t_{\lim }}}{\sqrt{t_{\lim }}} \sinh \left(\sqrt{t_{\lim }}\left(r-r_{0}\right)\right) d r \\
& =\frac{\ell\left(s_{i}\right) \sqrt{1-t_{\lim }}}{t_{\lim }}\left(\cosh \left(\sqrt{t_{\lim }}\left(-r_{0}\right)\right)-1\right) \\
& =\frac{\ell\left(s_{i}\right) \sqrt{1-t_{\lim }}}{t_{\lim }}\left(\cosh \left(\tanh ^{-1}\left(\sqrt{t_{\lim }}\right)\right)-1\right) \\
& =\frac{\ell\left(s_{i}\right) \sqrt{1-t_{\lim }}}{t_{\lim }}\left(\frac{1}{\sqrt{1-t_{\lim }}}-1\right) \\
& =\frac{\ell\left(s_{i}\right) \cdot 2 \pi / \ell\left(s_{i}\right)}{1-\left(2 \pi / \ell\left(s_{i}\right)\right)^{2}}\left(\frac{\ell\left(s_{i}\right)}{2 \pi}-1\right) \\
& =\frac{\ell\left(s_{i}\right)^{2}}{\ell\left(s_{i}\right)+2 \pi} .
\end{aligned}
$$

Therefore, for $t$ sufficiently close to $t_{\text {lim }}$, we obtain item (4).

\section{Sweepouts and generalized Heegaard splittings}

In this section, we review the definitions of strongly irreducible Heegaard splittings, and untelescoping for weakly reducible Heegaard splittings. We recall the definition of a sweepout, as well as bounded area sweepouts. For strongly irreducible Heegaard splittings, the existence of bounded area sweepouts follows from results announced by Pitts and Rubinstein [17]. When the Heegaard splitting is not strongly irreducible, we still obtain a bounded area sweepout after untelescoping.

None of the results in this section are original. However, since the ideas described here are gathered from many sources, we found it helpful to write down a unified exposition. 
Definition 3.1. A compression body $C$ is constructed by taking a closed, oriented (possibly disconnected) surface $S$ with no $S^{2}$ components, thickening it to $S \times[0,1]$, and attaching a finite number of 1 -handles to $S \times\{1\}$ in a way that makes the result connected. The negative boundary of $C$ is $\partial_{-} C:=S \times\{0\}$, and the positive boundary is $\partial_{+} C:=\partial C \backslash \partial_{-} C$.

A handlebody of genus $g$, constructed by attaching $g$ 1-handles to a 3-ball, is also considered a compression body. Its negative boundary is empty, and its positive boundary is the genus $g$ surface $\partial C$.

The spine of a compression body $C$ consists of the negative boundary $\partial_{-} C$, along with the core arcs of the attached 1-handles. In the special case where $C$ is a handlebody, a spine is any graph whose regular neighborhood is $C$. In either case, a compression body $C$ deformation retracts to its spine, and in fact, the complement of the spine in $C$ is homeomorphic to $\partial_{+} C \times(0,1)$.

Definition 3.2. A Heegaard splitting of a compact orientable 3-manifold $M$ is an expression $M=C_{1} \cup C_{2}$, where $C_{1}$ and $C_{2}$ are compression bodies glued along their positive boundaries. The surface

$$
\Sigma=C_{1} \cap C_{2}=\partial_{+} C_{1}=\partial_{+} C_{2}
$$

is called the Heegaard surface of the splitting $M=C_{1} \cup C_{2}$. We often speak of the Heegaard splitting and its surface interchangeably, as one determines the other.

A Heegaard splitting $M=C_{1} \cup C_{2}$ defines a sweepout. This is a map $f: M \rightarrow[-1,1]$, such that:

- $f^{-1}(-1)$ is a spine of $C_{1}$ and $f^{-1}(1)$ is a spine of $C_{2}$.

- For each $t \in(-1,1), f^{-1}(t)$ is a surface isotopic to $\Sigma=\partial_{+} C_{1}=\partial_{+} C_{2}$.

Writing $\Sigma_{t}=f^{-1}(t)$, we obtain a 1 -parameter family of surfaces isotopic to $\Sigma$, interpolating between the two spines.

Definition 3.3. A Heegaard splitting $M=C_{1} \cup C_{2}$ is called reducible if there are properly embedded discs $D_{i} \subset C_{i}$ that are essential (i.e., $\partial D_{i} \subset$ $\partial_{+} C_{i}$ is non-trivial on $\left.\partial_{+} C_{i}\right)$ and such that $\partial D_{1}=\partial D_{2} \subset \Sigma$. If $M$ is irreducible, the sphere $D_{1} \cup D_{2}$ must bound a 3 -ball, hence $\Sigma$ is obtained by adding extra handles inside the 3 -ball, a process called stabilization. Otherwise, if the splitting is not reducible, it is called irreducible.

Following Casson and Gordon [4], we say a Heegaard splitting is weakly reducible if there exist essential compression discs $D_{i} \subset C_{i}$ such that $\partial D_{1} \cap$ $\partial D_{2}=\emptyset$. It is strongly irreducible otherwise. 
Casson and Gordon showed that an irreducible manifold $M$ with a nonstabilized weakly reducible splitting must contain an incompressible surface [4]. Scharlemann and Thompson showed that in this setting, we may always cut $M$ along incompressible surfaces and obtain a strongly irreducible generalized Heegaard splitting [25]. Our description of these splittings follows Lackenby's paraphrase [13].

Definition 3.4. A generalized Heegaard splitting of a compact orientable 3-manifold $M$ is a decomposition of $M$ into submanifolds $W_{1}, \ldots, W_{m}$, for an even $m$, where each $W_{i}$ is a disjoint union of compression bodies, glued along their boundaries as follows. For each $0<i<m / 2$, we have

$$
\partial_{-} W_{2 i} \cap M^{\circ}=\partial_{-} W_{2 i+1} \cap M^{\circ}, \quad \text { and } \quad \partial_{+} W_{2 i}=\partial_{+} W_{2 i-1}
$$

(Here, the notation $M^{\circ}$ indicates the interior of $M$.) The surface $F_{i}=W_{i} \cap$ $W_{i+1}$ is called an even or odd surface, depending on the parity of $i$. By construction, each component of the odd surface $F_{2 i-1}$ is a Heegaard surface of the corresponding component of $\left(W_{2 i-1} \cup W_{2 i}\right)$.

Using the work of Casson and Gordon [4], Scharlemann and Thompson proved that one may start with a 3 -manifold $M$ and an irreducible splitting $\Sigma$, and construct a generalized Heegaard splitting with the following properties:

1) Every component of every odd surface $F_{2 i-1}$ is a strongly irreducible Heegaard surface for the component of $\left(W_{2 i-1} \cup W_{2 i}\right)$ that contains it.

2) Every even surface $F_{2 i}$ is incompressible, with no 2-sphere components.

3) Every $F_{i}$ satisfies $\chi(\Sigma) \leq \chi\left(F_{i}\right) \leq 0$.

A generalized Heegaard splitting with these properties is called thin.

The process of constructing a generalized Heegaard splitting is called untelescoping, and the inverse process (which recovers $\Sigma$ ) is called amalgamation. We describe the amalgamation process briefly, since we will need it below.

Choose an even surface in the generalized splitting, say $F_{2}$. By Definition 3.4, a component $S_{2} \subset F_{2}$ is the negative boundary of a compression body $C_{2} \subset W_{2}$ and a compression body $C_{3} \subset W_{3}$. By Definition 3.1, $C_{2}$ is constructed by attaching 1-handles to a surface $S \times\{1\}$, which is parallel to $S_{2}=S \times\{0\}$. Extend these 1 -handles through $S \times[0,1]$, and attach 
them directly to $S_{2}$. Similarly, extend the 1-handles of the compression body $C_{3} \subset W_{3}$, to attach them directly to $S_{2} \subset F_{2}$. This can be done while keeping the attaching discs disjoint from the attaching discs of the 1-handles in $C_{2}$. See [13, Figure 12].

If we perform this construction for every component of $F_{2}$, the resulting surface $F_{2}^{\prime}$, obtained from $F_{2}$ by attaching annuli, is now a Heegaard surface of $W_{1} \cup \cdots \cup W_{4}$. In other words, we have obtained a generalized Heegaard splitting with fewer pieces. Continuing in this manner, we amalgamate all the pieces and obtain a Heegaard surface for $M$. Although there are various choices involved this procedure (choosing handle structures on the compression bodies, choosing an order in which to amalgamate), amalgamation is guaranteed to recover the original Heegaard surface $\Sigma \subset M$. See [13, Proposition 3.1].

Our goal is to work with generalized Heegaard splittings in the context of a negatively curved metric, as constructed in Section 2.

Definition 3.5. Let $M$ be an orientable Riemannian 3-manifold. An orientable surface $F \subset M$ is called almost minimal if $F$ is either a minimal surface, or is the boundary of an $\varepsilon$-neighborhood of a non-orientable minimal surface $F^{\prime}$.

We will use the following result on essential surfaces and minimal surfaces; see [8, 15, 26].

Lemma 3.6. Let $M$ be a negatively curved Riemannian 3-manifold, whose boundary (if any) consists of minimal surfaces. Then

1) A connected, orientable, essential surface $F \subset M$ is isotopic to an almost minimal surface, as in Definition 3.5.

2) If $F$ and $G$ are disjoint, connected, non-parallel essential surfaces, then their almost-minimal representatives are also disjoint.

In particular, if $\left\{W_{1}, \ldots, W_{m}\right\}$ form a thin generalized Heegaard splitting for $M$, one may isotope the $W_{j}$ into a position where each piece $M_{i}=W_{2 i-1} \cup W_{2 i}$ has minimal boundary. A boundary surface of $M_{i}$ may be part of the original boundary of $M$, or it may result from cutting along a minimal surface corresponding to an even $F_{i}$, as in Lemma 3.6. (Note that if $F_{i}$ is isotopic to the orientable double cover of a non-orientable minimal surface $F_{i}^{\prime}$, then cutting along $F_{i}^{\prime}$ is equivalent to cutting along $F_{i}$, and produces orientable, minimal boundary.) 
Every minimal surface in $M$ satisfies an upper bound on area, related to its curvature.

Lemma 3.7. Let $M$ be an orientable Riemannian 3-manifold whose sectional curvatures are bounded above by $\kappa_{\max }<0$. Let $S \subset M$ be a minimal surface. Then

$$
\operatorname{area}(S) \leq 2 \pi \chi(S) / \kappa_{\max }
$$

Proof. This follows from the Gauss-Bonnet theorem, combined with the properties of minimal surfaces. Let $\lambda_{1}, \lambda_{2}$ denote the principal normal curvatures at a point $x \in S$, let $K_{x}$ denote the sectional curvature of $M$ along $S$, and let $\kappa$ denote the Gaussian curvature of $S$ at $x \in S$. Then the minimality of $S$ implies that $\lambda_{1}=-\lambda_{2}$ hence

$$
\kappa=K_{x}+\lambda_{1} \lambda_{2} \leq K_{x} \leq \kappa_{\max }
$$

Integrating over $S$, we obtain

$$
\kappa_{\max } \operatorname{area}(S)=\int_{S} \kappa_{\max } d A \geq \int_{S} \kappa d A=2 \pi \chi(S),
$$

where the last equality is the Gauss-Bonnet theorem. Dividing the previous equation by $\kappa_{\max }$ gives the desired statement.

Note that if $F$ is the orientable double cover of a non-orientable minimal surface $F^{\prime}$, as in Definition 3.5, then choosing a sufficiently small $\varepsilon$-neighborhood ensures that area $(F)$ satisfies a bound arbitrarily close to (3.1). This will be useful for Heegaard surfaces.

The following lemma is essentially a reformulation of an announced result by Pitts and Rubinstein [17]. As mentioned in the introduction, a complete proof of their result has not yet appeared.

Lemma 3.8. Let $M$ be an orientable Riemannian 3-manifold whose noncompact ends (if any) are isometric to horospherical cusp neighborhoods, and whose boundary (if any) consists of minimal surfaces. Suppose the sectional curvatures of $M$ are bounded above by $\kappa_{\max }<0$. Let $\Sigma$ be a strongly irreducible Heegaard surface for $M$. Then, for any $\zeta \in(0,1)$, there is a sweepout of $M$ corresponding to $\Sigma$, such that every level surface $\Sigma_{t}$ satisfies

$$
\operatorname{area}\left(\Sigma_{t}\right) \leq \frac{2 \pi \chi(\Sigma)}{\zeta \kappa_{\max }}
$$


Proof. As described in Definition 3.2, the Heegaard surface $\Sigma$ specifies a sweepout of $M$. In any choice of sweepout, there is a surface $\Sigma_{t}$ of maximal area. Let $A$ be the infimum of these maximal areas, over all sweepouts corresponding to $\Sigma$. The number $A$ is called a minimax value.

The work of Pitts and Rubinstein implies that there is a minimal surface $F \subset N$ whose area is the minimax value $A$ if $F$ is orientable, or $A / 2$ if $F$ is non-orientable [17]. Furthermore, $\Sigma$ is constructed by taking an almost minimal surface corresponding to $F$, and then possibly attaching a single tube. Both of these operations (taking the boundary of an $\varepsilon$-neighborhood, attaching a tube) can be achieved while increasing the area $A$ by an arbitrarily small amount. Thus, Lemma 3.7 guarantees that for any $\zeta \in(0,1)$, there is a sweepout whose level surfaces satisfy

$$
\operatorname{area}\left(\Sigma_{t}\right) \leq 2 \pi \chi(\Sigma) / \zeta \kappa_{\max }
$$

as desired.

\section{Core of Dehn filling and Heegaard surfaces}

The goal of this section is to prove Theorem 1.3, which was stated in the introduction. Most of the work here goes into proving the following, slightly simpler statement.

Theorem 4.1. Let $M$ be an orientable Riemannian 3-manifold whose noncompact ends (if any) are isometric to horospherical cusp neighborhoods, and whose boundary (if any) consists of minimal surfaces. Suppose the sectional curvatures of $M$ are bounded above by $\kappa_{\max }<0$. Let $\left\{W_{1}, \ldots, W_{m}\right\}$ be a thin generalized Heegaard splitting of $M$, with separating surfaces $F_{i}=W_{i} \cap$ $W_{i+1}$.

Let $V$ be a solid torus in $M$, such that the metric on $V$ is submersible. If a cross-sectional disc $D$ of $V$ satisfies

$$
\operatorname{area}(D)>\frac{2 \pi \chi\left(F_{i}\right)}{\kappa_{\max }} \quad \forall i,
$$

then the core curve $\gamma$ of $V$ is isotopic into one of the odd surfaces $F_{i}$.

The statement of Theorem 4.1 is similar to Theorem 1.3, with the single difference that a Heegaard surface $\Sigma$ has been replaced by a thin generalized Heegaard splitting. In our applications, this will be the generalized splitting obtained by untelescoping $\Sigma$. Furthermore, by amalgamating the generalized 
splitting to recover $\Sigma$, we will see that Theorem 1.3 follows quickly from this statement.

For the remainder of the section, we will use the definitions and notation of Theorem 4.1. In particular, $M$ will denote a negatively curved 3-manifold, with a generalized Heegaard splitting $\left\{W_{1}, \ldots, W_{m}\right\}$. As in the theorem, $V$ will denote a submersible solid torus in $M$, whose cross-sectional disc satisfies (4.1).

The proof of Theorem 4.1 is essentially due to Breslin, and follows the same line of argument as in his paper [3]. We need to make slight modifications to his argument to accommodate manifolds with boundary, generalized Heegaard splittings, and the metric of variable negative curvature. However, the spirit of the argument is the same. Where our argument differs from his, we walk through the details carefully.

The proof breaks down into the following claims:

(1) The core curve $\gamma$ of $V$ is isotopic into the complement of the even surfaces. This means that we can work with a single odd surface $S=F_{i}$, which is strongly irreducible in its component. We will show this in Lemma 4.3.

(2) The Heegaard surface $S=F_{i}$ contains a simple loop homotopic to $\gamma^{n}$ for some $n$. The geometric proof of this claim, based on [3, Lemma 2], appears in Lemma 4.4 .

(3) There is an embedded annulus $A \subset M$, such that one component of $\partial A$ is on $S$ and the other component of $\partial A$ is on $\partial N(\gamma)$, a tubular neighborhood of $\gamma$. The argument is nearly the same as that of $[3$, Lemma 1], and is recalled in Lemma 4.6.

(4) The loop $\partial A \cap \partial N(\gamma)$ is isotopic to $\gamma$, hence $\gamma$ is isotopic into $S$ through $A$. The proof is identical to [3, Lemma 5], which we restate in Lemma 4.7.

The following lemma is useful for both incompressible surfaces and strongly irreducible Heegaard surfaces. The proof is inspired by [3, Claim 1].

Lemma 4.2. Let $M$ and $V$ be as in Theorem 4.1. Suppose that $F$ is a compact, orientable surface embedded in $M$, such that

$$
\operatorname{area}(F)<\operatorname{area}(D)
$$

where $D$ is the cross-sectional disc in $V$. Then $V \backslash F$ contains a closed curve that is essential in $V$. 
Proof. If $F \cap V$ contains a closed curve that is essential in $V$, we may homotope this curve to one side of $F$, into $V \backslash F$, satisfying the conclusion of the lemma. Thus, we may assume that $F \cap V$ does not contain an essential curve.

Consider lifts of $F \cap V$ in $\widetilde{V}$, the universal cover of $V$. Since $F \cap V$ does not contain an essential curve, there is a lift $\widetilde{F}$ of $F \cap V$ in $\widetilde{V}$ that is isometric to $F \cap V$. Because $\widetilde{V}$ is a ball, any connected component of $\widetilde{F}$ must separate $\widetilde{V}$. We want to show that the two ends of $\widetilde{V}$ are contained in the same component of $\widetilde{V} \backslash \widetilde{F}$.

Let $D$ be a cross-sectional disc of $\widetilde{V}$. By Definition 1.2 , there is a Riemannian submersion $f: \widetilde{V} \rightarrow D$. Because Riemannian submersions reduce area, and area $(F \cap V)<\operatorname{area}(D)$, the projection of $\widetilde{F}$ must miss some point $x \in D$. Then the fiber $\{x\} \times \mathbb{R} \subset \widetilde{V}$ is disjoint from $\widetilde{F}$, hence no component of $\widetilde{F}$ can separate the ends of $\widetilde{V}$.

The lift of a meridian disc to $\widetilde{V}$ consists of a disjoint family of discs $D_{j}$, for $j \in \mathbb{Z}$, where a deck transformation maps $D_{j}$ to $D_{j+1}$. For a natural number $n$, let $R=R_{n}$ be the component of $\widetilde{V} \backslash\left(D_{-n} \cup D_{n}\right)$ that has compact closure. Because $F$ is compact, there are finitely many lifts $\widetilde{F}_{1}, \ldots, \widetilde{F}_{k}$ of $F \cap V$ that intersect the closure of $R$. Each component $\widetilde{F}_{i}$ splits $R$ into two pieces, one of which does not separate the ends of $\widetilde{V}$. Thus the set $\widetilde{V} \backslash\left(\widetilde{F}_{1} \cup \cdots \cup \widetilde{F}_{k}\right)$ contains a component that intersects both $D_{-n}$ and $D_{n}$. In other words, there is a path from $D_{-n}$ to $D_{n}$ that is disjoint from the complete preimage of $F \cap V$. Since $n$ was arbitrary, it follows that some component of the preimage of $V \backslash F$ in $\widetilde{V}$ has non-compact closure. The projection of this component to $V$ must contain a non-trivial curve.

Using Lemma 4.2, we can show that the core curve $\gamma$ of $V$ is disjoint from the even, incompressible surfaces in the generalized Heegaard splitting.

Lemma 4.3. Let $M$ and $V$ be as in Theorem 4.1. Let $F=F_{2} \cup F_{4} \cup \cdots \cup$ $F_{m-2}$ be the union of the even surfaces in the generalized Heegaard splitting of $M$, and suppose (following Lemma 3.6) that each even $F_{i}$ has been isotoped to be almost minimal in M. Suppose that the cross-sectional disc of $V$ satisfies

$$
\operatorname{area}(D)>2 \pi \chi\left(F_{i}\right) / \kappa_{\max } \quad \forall i
$$

Then some component $V^{\prime}$ of $V \backslash F$ is a solid torus, isotopic in $M$ to $V$ itself. Furthermore, each component of $V \backslash V^{\prime}$ is a trivial I-bundle over a subsurface of $\partial V$. 
Proof. By Lemma 3.6, each even $F_{i}$ is isotopic to a minimal surface, or is the boundary of an $\varepsilon$-neighborhood of a non-orientable minimal surface. Then Lemma 3.7 implies that for sufficiently small $\varepsilon$, we can ensure the area of $F_{i}$ is bounded above by $2 \pi \chi\left(F_{i}\right) / \kappa_{\max }+\delta$, for arbitrarily small $\delta$. In particular, we have

$$
\operatorname{area}\left(F_{i}\right)<\operatorname{area}(D) \text {. }
$$

Consider the curves of intersection $F \cap \partial V$. If one of these curves is a meridian of $V$, it must bound a disc in some $F_{i}$, because $F_{i}$ is incompressible. Pass to an innermost disc in $F_{i}$, among all discs whose boundary is a meridian curve on $\partial V$. This disc must be a meridian of $V$, because $M$ is negatively curved, hence irreducible. However, then $V \backslash F$ cannot contain a curve that is essential in $V$, contradicting Lemma 4.2. Therefore, every curve of $F \cap \partial V$ is either trivial in $\partial V$, or else essential in $V$.

As a preliminary step for the proof, we isotope trivial curves of $F \cap \partial V$ off $V$, through balls, as follows. If any curve of $F \cap \partial V$ is trivial in $\partial V$, at least one such trivial curve must be innermost in $F$. This curve must bound a disc $D_{0} \subset F$ and a disjoint disc $D_{1} \subset \partial V$. Because $M$ is irreducible, we may isotope $D_{0}$ past $D_{1}$, to remove this curve of intersection. If any trivial curves remain, repeat this procedure with another innermost curve. After this sequence of isotopies, any remaining curves of $F \cap \partial V$ are essential in $V$.

Now, consider a component $A_{0}$ of $F \cap V$ (if any). Since each component of $F$ is essential in $M$, and every curve of $\partial A_{0}$ is essential in $V$, the component $A_{0}$ must itself be incompressible in $V$. Since $\pi_{1}\left(A_{0}\right) \hookrightarrow \pi_{1}(V) \cong \mathbb{Z}$ and $F$ is 2 -sided, this means $A_{0}$ is an annulus. As all annuli in a solid torus are boundary-parallel, $F \cap V$ consists entirely of boundary-parallel annuli. Each boundary-parallel annulus $A_{0}$ cuts off a boundary-parallel solid torus in $V$.

After removing all of these boundary-parallel pieces (if any), we find a component $V^{\prime}$ of $V \backslash F$ that is isotopic to $V$ itself. Now, undo the isotopies through balls that removed the trivial curves of intersection of $F \cap \partial V$ in the preliminary step of the proof. Each of these isotopies modifies $V^{\prime}$ by pushing a disc on the boundary into or out of $V^{\prime}$. In particular, these isotopies preserve the property that the component $V^{\prime}$ of $V \backslash F$ is isotopic to $V$ itself. They also preserve the property that each component of $V \backslash V^{\prime}$ is boundaryparallel.

Recall the thin generalized splitting $\left\{W_{1}, \ldots, W_{m}\right\}$ of $M$, with surfaces $F_{i}=W_{i} \cap W_{i+1}$. By Lemma 4.3, we know that there is a solid torus $V^{\prime} \subset V$, which is isotopic to $V$ itself, and is disjoint from every even surface $F_{2 i}$. This means that $V^{\prime}$ is contained in some submanifold $W_{2 i-1} \cup W_{2 i}$, in the 
complement of the even surfaces. For the rest of this section, we take $W$ to be the connected component of $W_{2 i-1} \cup W_{2 i}$ containing $V^{\prime}$.

The following lemma, and its proof, was inspired by [3, Lemma 2].

Lemma 4.4. Let $M$ and $V$ be as in Theorem 4.1, and let $V^{\prime}$ be as in Lemma 4.3. Let $S \subset F_{2 i-1}$ be a strongly irreducible Heegaard surface for the submanifold $W$ that contains $V^{\prime}$. Then, after an isotopy of $S$, there is a simple closed curve $\delta \subset S \cap V^{\prime}$ that is essential in $V^{\prime}$.

Proof. By Equation (4.1), the cross-sectional disc $D$ of $V$ satisfies the strict inequality area $(D)>2 \pi \chi(S) / \kappa_{\max }$. Thus, for some $\zeta \in(0,1)$ near 1 , we have

$$
\operatorname{area}(D)>2 \pi \chi(S) /\left(\zeta \kappa_{\max }\right)
$$

For this value of $\zeta$, Lemma 3.8 implies that there exists a sweepout $f: W \rightarrow$ $[-1,1]$ corresponding to $S$, such that every level surface $S_{t}$ satisfies

$$
\operatorname{area}\left(S_{t}\right) \leq 2 \pi \chi(\Sigma) /\left(\zeta \kappa_{\max }\right)<\operatorname{area}(D)
$$

Let $V^{\prime} \subset V \cap W$ be the solid torus guaranteed by Lemma 4.3, which is isotopic to $V$.

Claim 4.5. For every $t \in(-1,1)$, some component of $V^{\prime} \backslash S_{t}$ contains a closed curve that is essential in $V^{\prime}$.

Proof of Claim. By Equation (4.2) and Lemma 4.2, we know that some component of $V \backslash S_{t}$ contains a closed curve that is essential in $V$. What needs to be shown is that this curve can be taken to lie in $V^{\prime}$.

Let $G_{t}=S_{t} \cap V^{\prime}$. Since $\partial V^{\prime}$ consists of sub-surfaces of $\partial V$ and sub-surfaces of $F=F_{2} \cup F_{4} \cup \cdots \cup F_{m-2}$, and since $S_{t}$ is disjoint from $F$, we know that $\partial G_{t} \subset \partial V$, i.e., $G_{t}$ is properly embedded in $V$.

Since $V$ contains an essential closed curve in the complement of $S_{t}$, it also contains such a curve in the complement of $G_{t}$. Furthermore, by Lemma 4.3, $V \backslash V^{\prime}$ consists of trivial $I$-bundles over subsurfaces of $\partial V$. Thus, we may homotope the essential closed curve through the interior of $V$, away from these boundary-parallel pieces and into $V^{\prime}$.

For each $t \in(-1,1)$, let $H_{t}=f(S \times[-1, t)) \subset W$ and $J_{t}=f(S \times$ $(t, 1]) \subset W$. Note the closure of $H_{t}$ is the compression body below $S_{t}$, and 
the closure of $J_{t}$ is the compression body above $S_{t}$. Define

$E_{H}:=\left\{t \in(-1,1): H_{t} \cap V^{\circ}\right.$ contains a closed curve that is essential in $\left.V^{\prime}\right\}$, $E_{J}:=\left\{t \in(-1,1): J_{t} \cap V^{\circ}\right.$ contains a closed curve that is essential in $\left.V^{\prime}\right\}$.

By Claim 4.5, we know that every $t \in(-1,1)$ is contained in either $E_{H}$ or $E_{J}$.

Since each of $H_{t} \cap V^{\circ}$ and $J_{t} \cap V^{\circ}$ is open in $W$, we know that both $E_{H}$ and $E_{J}$ are open sets. Furthermore, for $t$ close to $-1, H_{t}$ is a small regular neighborhood of a spine of $W_{2 i-1}$; hence these values of $t$ must be contained in $E_{J}$. Similarly, for $t$ close to $1, J_{t}$ is a small regular neighborhood of a spine of $W_{2 i}$, hence these values of $t$ must be contained in $E_{H}$. Since both $E_{H}$ and $E_{J}$ are open and non-empty, and their union $(-1,1)$ is connected, it follows that $E_{H} \cap E_{J} \neq \emptyset$.

Let $r \in E_{H} \cap E_{J}$. Then, by construction, $H_{r} \cap V^{\circ}$ contains an essential loop that we call $\alpha_{H}$, and $J_{r} \cap V^{\circ}$ contains an essential loop that we call $\alpha_{J}$. There exist integers $n, m$ so that $\left(\alpha_{H}\right)^{n}$ is homotopic to $\left(\alpha_{J}\right)^{m}$ in $V$, and thus there is an immersed annulus $A$ in $V^{\prime}$ with boundary components $\left(\alpha_{H}\right)^{n}$ and $\left(\alpha_{J}\right)^{m}$. This implies that some loop $A \cap S_{r}$ is non-trivial in $A$, and hence, by taking a sub-loop, if necessary, there exists an embedded essential loop in $A \cap S_{r}$, which must therefore be essential in $V^{\prime}$. Since the sweepout surface $S_{r}$ is isotopic to $S$, we are done.

The rest of the proof follows from two lemmas, whose topological proofs are due to Breslin. We need only check that his proofs carry through in our setting. The first is [3, Lemma 1].

Lemma 4.6. Let the submanifold $W$ and the Heegaard surface $S \subset F_{2 i-1}$ be as in Lemma 4.4. Let $\gamma$ be the core curve of $V$, as in Theorem 4.1. Then, possibly after isotoping $\gamma$, there is a regular neighborhood $N(\gamma)$ and an embedded annulus $A$ in $W \backslash N(\gamma)$ with $\partial A=\alpha \cup \alpha^{\prime}$, where $\alpha$ is a simple essential non-meridional loop in the boundary of $N(\gamma)$ and $\alpha^{\prime} \subset S$.

Proof. By Lemma 4.4, we may isotope $S$ so that $S \cap V^{\prime}$ contains a simple loop $\alpha$ that is essential in $V^{\prime}$, where $V^{\prime} \subset V$ is the solid torus of Lemma 4.3. Recall the sweepout $f: W \rightarrow[-1,1]$, which was constructed in the proof of Lemma 4.4. This map has the property that $f^{-1}(t)=S_{t}$ is isotopic to $S$ for each $t \in(-1,1)$, while the sets $f^{-1}( \pm 1)$ consist of the spines of the two compression bodies.

The next step is to use the Rubinstein-Scharlemann graphic. For closed manifolds, the graphic is defined in [22]. However, we need to use the 
Rubinstein-Scharlemann graphic for manifolds with boundary, as in [23]. In particular, one modification is that we only consider the part of the sweepout that runs from $S_{-1+\epsilon}$ to $S_{(1-\epsilon)}$, for some $\epsilon>0$ sufficiently small. This way, we avoid problems as the sweepout meets the boundary of $W$.

Let $g: V^{\prime} \rightarrow[0,1]$ be a smooth function that gives a sweepout of the solid torus. That is, for all $s \in(0,1), g^{-1}(s)$ is a surface isotopic to $\partial V^{\prime}$, while $g^{-1}(1)=\partial V^{\prime}$, and $g^{-1}(0)$ is a closed curve isotopic to $\gamma$, the core of $V$. Isotope $\gamma$ to be $g^{-1}(0)$. We will consider the function $g_{t}=\left.g\right|_{S_{t} \cap V^{\prime}}$, and apply work of Cerf [5] to isotope $f$ and $g$ so that $g_{t}$ is Morse for all but finitely many $t$, and near-Morse otherwise. The Rubinstein-Scharlemann graphic $G$ is the set of points $(t, s) \in[-1,1] \times[0,1]$ such that $s$ is a critical value of $g_{t}$. While care must be taken for applications of the graphic for manifolds with boundary (compare [22, 23]), to apply Breslin's proof we only need the result from Cerf theory that if $\left(t_{1}, s_{1}\right)$ and $\left(t_{2}, s_{2}\right)$ are in the same component of $([-1+\epsilon, 1-\epsilon] \times[0,1]) \backslash G$, then the surface $S_{t_{1}}$ is isotopic to $S_{t_{2}}$ via an isotopy that takes the loops in $g_{t_{1}}^{-1}\left(s_{1}\right)$ to the loops in $g_{t_{2}}^{-1}\left(s_{2}\right)$.

Using the graphic described above, we obtain [3, Lemma 4]. According to that lemma, one of two conclusions must hold for some $t \in(-1,1)$ :

1) $S_{t} \cap \partial V^{\prime}$ contains a loop that is essential and non-meridional on $\partial V^{\prime}$, or

2) $S_{t} \cap V^{\prime}$ does not contain an essential loop of $S_{t}$.

The proof of [3, Lemma 4] works verbatim with the slight modification to the sweepout that was mentioned above. In addition to the RubinsteinScharlemann graphic, it uses Scharlemann's No Nesting Lemma [24]. Note that this lemma applies equally well to 3 -manifolds with and without boundary.

In case (1) above, we are essentially done with the proof of Lemma 4.6, as follows. Let $N(\gamma)=g^{-1}[0,1-\epsilon]$ be a solid torus slightly inside $V^{\prime}$. Let $\alpha^{\prime} \subset S_{t} \cap g^{-1}(1)$ be the loop guaranteed by conclusion (1), and let $\alpha$ be the projection of $\alpha^{\prime}$ to $\partial N(\gamma)=g^{-1}(1-\epsilon)$. Then the product annulus between $\alpha$ and $\alpha^{\prime}$ satisfies the lemma.

In case (2) above, there is a $t \in(-1,1)$ so that $S_{t} \cap V^{\prime}$ does not contain an essential loop of $S_{t}$, and thus each loop in $S_{t} \cap \partial V^{\prime}$ bounds a disc in $S_{t}$. By Lemma 4.4, another sweepout surface $S_{r}$ has the property that $S_{r} \cap V^{\prime}$ contains a simple loop $\alpha_{r}$ that is essential in $V^{\prime}$. Since $S_{r}$ and $S_{t}$ are isotopic, there is an embedded annulus $A$ with one boundary component equal to $\alpha_{r} \subset S_{r}$ and the other equal to some $\alpha_{t} \subset S_{t}$. 
Because all loops of $S_{t} \cap \partial V^{\prime}$ bound discs on $S_{t}$, we may isotope $A$ to avoid these discs, hence $\alpha_{t}$ is disjoint from $S_{t} \cap \partial V^{\prime}$. The loop $\alpha_{t}$ must therefore be contained in the interior of $V^{\prime}$ or disjoint from $V^{\prime}$. Because $S_{t} \cap V^{\prime}$ contains no essential loop of $S_{t}$, and because $\alpha_{t}$ is isotopic to the essential loop $\alpha_{r}$, we must have $\alpha_{t}$ in $W \backslash V^{\prime}$. Isotope $A$ slightly, if necessary, so that $\alpha_{r}$ is contained in the interior of $V^{\prime}$. Then $A$ is an annulus embedded in $W$ with $\partial A=\alpha_{r} \cup \alpha_{t}$ with $\alpha_{r} \subset V^{\prime}$ and $\alpha_{t} \subset\left(W \backslash V^{\prime}\right)$. Thus $A$ meets $\partial V^{\prime}$ in an essential loop $\alpha_{t}^{\prime}$. Use the embedded annulus bounded by $\alpha_{t}$ and $\alpha_{t}^{\prime}$ to isotope $S_{t}$ so that $S_{t} \cap V^{\prime}$ contains a loop that is essential and non-meridional in $\partial V^{\prime}$. This puts us back into case (1), hence the proof is complete.

We have seen that there is an embedded annulus in $W$ with one boundary component on a neighborhood of $\gamma$ and one on our Heegaard surface $S=F_{2 i-1}$. This annulus must wrap around some power of $\gamma$. The final step toward proving Theorem 4.1 is to show that in fact, we may take the annulus to wrap exactly once around $\gamma$.

Lemma 4.7. Let $W$ be a 3-manifold with Heegaard surface $S$, and let $\gamma$ be a simple loop in $W$ with regular neighborhood $N(\gamma)$. Let $\alpha$ be an essential non-meridional loop in $N(\gamma)$, and $\alpha^{\prime}$ a loop in $S$. If there is an embedded annulus $A \subset W$ disjoint from $N(\gamma)$, with boundary $\alpha \cup \alpha^{\prime}$, then $\gamma$ is isotopic into $S$.

Proof. This is [3, Lemma 5], and Breslin's proof goes through verbatim. We note that his proof uses a thin position argument of Schultens [27], modified slightly by Breslin. The argument holds for manifolds with or without boundary, and indeed Schultens' original application concerned manifolds with boundary. Hence, we refer the reader to [3, Lemma 5] for the proof.

The referee informs us that Lemma 4.7 can also be proved via a straightforward application of the Daisy Lemma [10].

Proof of Theorem 4.1. Recall that $S \subset F_{2 i-1}$ is a component of one of the odd surfaces in a generalized Heegaard splitting of $M$. Now, the theorem follows by Lemmas 4.6 and 4.7 .

The above results also give a quick proof of Theorem 1.3, which was stated in the introduction.

Proof of Theorem 1.3. Let $\Sigma$ be a genus $g$ Heegaard surface of $M$. If $\Sigma$ is irreducible, then, as described in Section 3, we may untelescope $\Sigma$ to a thin 
generalized Heegaard splitting $\left\{W_{1}, \ldots, W_{m}\right\}$. By property (3) of thinness, the cross-sectional disc $D$ of the submersible solid torus $V$ satisfies

$$
\operatorname{area}(D)>\frac{2 \pi \chi(\Sigma)}{\kappa_{\max }} \geq \frac{2 \pi \chi\left(F_{i}\right)}{\kappa_{\max }} \quad \forall i .
$$

Thus, by Theorem 4.1, the core curve $\gamma$ of $V$ is isotopic into some odd surface $F_{2 i-1}$.

Now, we assume $\gamma \subset F_{2 i-1}$, and amalgamate the generalized Heegaard splitting to recover $\Sigma$. At certain times during the amalgamation process, we will need to attach handles to a partially amalgamated surface containing $\gamma$. Each time we do this, a small isotopy ensures that $\gamma$ is disjoint from the discs along which we attach handles. Thus, after the amalgamation is complete, we have $\gamma \subset \Sigma$.

Meanwhile, if $\Sigma$ is reducible, then we destabilize $\Sigma$ to an irreducible Heegaard surface $\Sigma^{\prime}$ of genus $h<g$, apply the above argument to $\Sigma^{\prime}$, and then stabilize back to genus $g$. Since stabilizations are unique, this approach recovers the desired result for $\Sigma$.

\section{Assembling the pieces}

In this section, we complete the proof of Theorem 1.1. The following lemma will permit us to apply the results of Section 4 .

Lemma 5.1. Let $C_{1}, \ldots, C_{k}$ be disjoint cusp neighborhoods of a hyperbolic 3-manifold $X$, and let $s_{i}$ be a slope on cusp $C_{i}$. Suppose that the shortest slope length is $\ell_{\min }>2 \pi(2 g-1)$, for some $g \geq 2$. Then the Dehn filling $M=X\left(s_{1}, \ldots, s_{k}\right)$ admits a negatively curved metric as in Theorem 2.1, with curvatures bounded by $\kappa_{\max }<0$, such that the area of a cross-sectional disc of every Dehn filling solid torus satisfies

$$
\operatorname{area}(D)>\frac{2 \pi(2 g-2)}{\left|\kappa_{\max }\right|} .
$$

Proof. If $\ell_{\min }>2 \pi(2 g-1)$, then Theorem 2.1 implies that for every $\zeta \in(0,1)$, the Dehn filled manifold $M$ admits a Riemannian metric with sectional curvatures bounded by $\kappa_{\max }=\zeta\left(4 \pi^{2} / \ell_{\min }{ }^{2}-1\right)$, and with crosssectional discs of area at least $\zeta \ell_{\min }^{2} /\left(\ell_{\min }+2 \pi\right)$, where

$$
\ell_{\min }>2 \pi(2 g-1) \text {. }
$$


Since the above inequality is strict, we may find $\zeta$ near 1 such that $\ell_{\min }>$ $\left(2 \pi / \zeta^{2}\right)\left(2 g-2+\zeta^{2}\right)$. Select this value of $\zeta$ for the application of Theorem 2.1 .

Then for any cross-sectional disc $D$,

$$
\begin{aligned}
\operatorname{area}(D) & \geq \frac{\zeta \ell_{\min }^{2}}{\ell_{\min }+2 \pi}=\frac{\zeta\left(\ell_{\min }-2 \pi\right)}{1-4 \pi^{2} / \ell_{\min }{ }^{2}} \geq \frac{\zeta^{2}\left(\ell_{\min }-2 \pi\right)}{\left|\kappa_{\max }\right|} \\
& >\frac{\zeta^{2}\left(\frac{2 \pi}{\zeta^{2}}\left(2 g-2+\zeta^{2}\right)-2 \pi\right)}{\left|\kappa_{\max }\right|}=\frac{2 \pi(2 g-2)}{\left|\kappa_{\max }\right|} .
\end{aligned}
$$

Proof of Theorem 1.1. Let $\Sigma$ be a Heegaard surface of genus $g$ for the Dehn filled manifold $M=X\left(s_{1}, \ldots, s_{k}\right)$. By Theorem 2.1, $M$ admits a metric with sectional curvatures bounded by $\kappa_{\max }<0$, which means $g \geq 2$. By Lemma 5.1 , the area of a cross-sectional disc of each Dehn filling solid torus satisfies $\operatorname{area}(D)>2 \pi \chi(\Sigma) / \kappa_{\max }$. Note that the solid tori constructed in Theorem 2.1 are submersible, as desired. Thus, by Theorem 1.3, we conclude that each core $\gamma_{i}$ of the $i^{\text {th }}$ solid torus is isotopic into $\Sigma$, as required for conclusion (1).

We will prove conclusion (2) by induction on $k$. That is, let $\Sigma$ be a Heegaard surface for $M=X\left(s_{1}, \ldots, s_{k}\right)$. In the following argument, we will show that the core $\gamma_{k}$ of the $k^{\text {th }}$ solid torus can be isotoped off $\Sigma$ in such a way that $\Sigma$ becomes a Heegaard surface for $M \backslash \gamma_{k} \cong X\left(s_{1}, \ldots, s_{k-1}\right)$. This argument works for arbitrary $k$. Hence by induction, $\Sigma$ becomes a Heegaard surface for $X$.

In the following argument, we may also assume without loss of generality that $\Sigma$ is irreducible. Otherwise, as in the proof of Theorem 1.3, we simply destabilize $\Sigma$ to an irreducible surface $\Sigma^{\prime}$, apply the argument to $\Sigma^{\prime}$, and stabilize at the end to recover $\Sigma$.

With these preliminaries out of the way, we assume that $\Sigma$ is irreducible in $X\left(s_{1}, \ldots, s_{k}\right)$, and untelescope $\Sigma$ to a thin generalized Heegaard splitting $\left\{W_{1}, \ldots, W_{m}\right\}$. Let $V=V_{k}$ be the $k^{\text {th }}$ Dehn filling solid torus, with the negatively curved metric of Theorem 2.1. By Lemma 5.1, the cross-sectional disc $D$ of $V$ satisfies

$$
\operatorname{area}(D)>\frac{2 \pi \chi(\Sigma)}{\kappa_{\max }} \geq \frac{2 \pi \chi\left(F_{i}\right)}{\kappa_{\max }},
$$

for each surface $F_{i}$ of the generalized splitting. Thus, by Theorem 4.1, the core curve $\gamma_{k}$ of $V=V_{k}$ is isotopic into some odd surface $F_{2 i-1}$. Recall that $S=F_{2 i-1}$ is a strongly irreducible Heegaard surface of the submanifold $W=W_{2 i-1} \cup W_{2 i}$. 
After $\gamma_{k}$ has been isotoped into $S$, consider the surface $S \backslash N\left(\gamma_{k}\right) \subset W$, where $N\left(\gamma_{k}\right)$ is a small tubular neighborhood of $\gamma_{k}$ contained in $W$. Let $\left\{D_{1}, \ldots, D_{n}\right\}$ be a collection of disjoint, non-parallel compression discs for $S \backslash N\left(\gamma_{k}\right)$ in $W$, which is maximal with respect to inclusion. Since $S$ is strongly irreducible, all of the $D_{j}$ must be contained in the same compression body, say $W_{2 i-1}$. Let $S^{\prime} \subset W_{2 i-1}$ be the surface obtained after compressing $S \backslash N\left(\gamma_{k}\right)$ along all of the $D_{j}$.

Claim 5.2. Each component of $S^{\prime}$ is either a sphere, a closed surface parallel to $\partial_{-} W_{2 i-1}$, or an annulus parallel to $N\left(\gamma_{k}\right)$.

Proof of claim. First, suppose that a component of $S^{\prime}$ is closed and not a 2 -sphere. Since we have compressed $S \backslash N\left(\gamma_{k}\right)$ along a maximal collection of discs, this component must be incompressible in $W_{2 i-1}$. However, the only incompressible surfaces in a compression body are parallel to the negative boundary $\partial_{-} W_{2 i-1}$.

Next, suppose that a component of $S^{\prime}$ is a surface with boundary, and call this component $R$. Since $\partial\left(S \backslash N\left(\gamma_{k}\right)\right)$ consists of two curves, both parallel to $\gamma_{k}, \partial R$ must be a union of one or two curves parallel to $\gamma_{k}$. Since the surface $S$ has genus at most $g$, and we obtained $R$ by cutting $S$ along $\gamma_{k}$ and then compressing, the genus of $R$ is at most $g-1$.

Suppose, for a contradiction, that $R$ is an essential surface in $W \backslash N\left(\gamma_{k}\right)$ : that is, not an annulus parallel to $N\left(\gamma_{k}\right)$. Then, since $\partial W=F_{2 i-2} \cup F_{2 i}$ is a union of incompressible surfaces, the component $R$ must also be essential in $X\left(s_{1}, \ldots, s_{k-1}\right)$. Isotope the cores $\gamma_{1}, \ldots, \gamma_{k-1}$ until they intersect $R$ minimally, and let $R^{\circ}=R \backslash\left(\gamma_{1} \cup \cdots \cup \gamma_{k-1}\right)$.

We claim that $R^{\circ}$ must be incompressible in the original cusped hyperbolic manifold $X$. To see this, suppose that an essential curve $\alpha \subset R^{\circ}$ bounds a compression disc $D_{0} \subset X$. By passing to an innermost sub-disc if needed, we may assume that the interior of $D_{0}$ is disjoint from $R$. However, $R$ is incompressible in $X\left(s_{1}, \ldots, s_{k-1}\right)$, hence $\alpha$ also bounds a disc $D_{1} \subset R$. Since $\alpha=\partial D_{1}$ is an essential curve in $R^{\circ}$, the disc $D_{1}$ must be punctured two or more times by the $\gamma_{i}$. On the other hand, $D_{0}$ is disjoint from all the cores $\gamma_{i}$, and has the same boundary as $D_{1}$. Isotoping these cores through the ball co-bounded by $D_{0}$ and $D_{1}$, past $D_{1}$, will reduce the intersection number between $R$ and $\gamma_{1} \cup \cdots \cup \gamma_{k-1}$, contradicting the construction of $R^{\circ}$. Therefore, $R^{\circ}$ is incompressible in $X$.

Recall that by construction, $R^{\circ}$ has a boundary component on $N\left(\gamma_{k}\right)$. Thus, if $R^{\circ}$ is a boundary-parallel annulus, then so is $R$, a contradiction. Thus $R^{\circ}$ is incompressible and boundary-incompressible in $X$. 
Now, remove the horospherical cusps $C_{1}, \ldots, C_{k}$ from both $X$ and $R^{\circ}$, and consider $\partial R^{\circ}$, which is a union of $b$ closed curves on $\partial C_{1}, \ldots, \partial C_{k}$. The curves of $\partial R$, which run parallel to $\gamma_{k}$, must be one or two longitudes of the filling slope $s_{k}$. Meanwhile, every other curve of $\partial R^{\circ}$ is a meridian of some $\gamma_{j}$ (for $1 \leq j \leq k-1$ ), hence is in the isotopy class of the filling slope $s_{j}$.

By the hypotheses of Theorem 1.1, the shortest longitude of $s_{k}$ has length $\ell\left(\lambda_{k}\right)>6(2 g-3)$. Each filling slope $s_{j}$ for $1 \leq j \leq k-1$ also has length

$$
\ell\left(s_{j}\right)>2 \pi(2 g-1)>6(2 g-1)>6(2 g-3) .
$$

We conclude that the total length $\ell\left(\partial R^{\circ}\right)$ of all the curves of $\partial R^{\circ}$ must satisfy

$$
b \cdot 6(2 g-3)<\ell\left(\partial R^{\circ}\right) \leq 6\left|\chi\left(R^{\circ}\right)\right| \leq 6(2(g-1)+b-2)=6(2 g+b-4),
$$

where the second inequality is a theorem of Agol [1, Theorem 5.1] and Lackenby [11, Lemma 3.3]. Comparing the first and last terms, we obtain

$$
\begin{aligned}
b \cdot(2 g-3) & <2 g+b-4, \\
2 g b-2 g-4 b+4 & <0, \\
2(g-2)(b-1) & <0,
\end{aligned}
$$

which is a contradiction since $g \geq 2$ and $b \geq 1$. This contradiction proves the claim.

Recall that we obtained $S^{\prime}$ from $S$ by compressing along a maximal collection of discs $\left\{D_{1}, \ldots, D_{n}\right\}$. By strong irreducibility of $S$, all of these discs are contained in the same compression body $W_{2 i-1}$. Because all of these discs are disjoint from $\gamma_{k}$, we may isotope $N\left(\gamma_{k}\right)$ into $W_{2 i-1}$ while staying disjoint from $\left\{D_{1}, \ldots, D_{n}\right\}$.

We claim that $W_{2 i-1} \backslash N\left(\gamma_{k}\right)$ is itself a compression body. This can be seen by building the compression body "downward" from its positive boundary $S=\partial_{+} W_{2 i-1}$. We thicken the surface $S$ into $S \times[0,1] \subset W_{2 i-1}$, and attach a 2-handle along each curve on $S \times\{0\}$ corresponding to $\partial D_{j}$ for each $j$. After attaching the 2-handles, the resulting negative boundary is exactly the surface $S^{\prime}$, with its two boundary curves joined together. By Claim 5.2, the surface obtained after attaching 2-handles consists of spheres, closed surfaces parallel to $\partial_{-} W_{2 i-1}$, and a single torus isotopic to $\partial N\left(\gamma_{k}\right)$. Thus, after capping off each 2-sphere with a 3 -ball, we obtain a 
compression body $W_{2 i-1}^{\prime}$, satisfying

$$
\partial_{+} W_{2 i-1}^{\prime}=\partial_{+} W_{2 i-1}=S, \quad \partial_{-} W_{2 i-1}^{\prime}=\partial_{-} W_{2 i-1} \cup \partial N\left(\gamma_{k}\right) .
$$

We have just shown that the core curve $\gamma_{k}$ may be isotoped off the generalized splitting surface, into $W_{2 i-1}$, in such a way that the submanifolds

$$
\left\{W_{1}, \ldots, W_{2 i}, W_{2 i-1}^{\prime}, W_{2 i+2}, \ldots, W_{m}\right\}
$$

form a thin generalized Heegaard splitting of $M \backslash \gamma_{k}=X\left(s_{1}, \ldots, s_{k-1}\right)$. After amalgamating this generalized splitting, we obtain a Heegaard surface $\Sigma^{\prime} \subset$ $M \backslash \gamma_{k}$.

Recall that, by [13, Proposition 3.1], amalgamation produces a unique Heegaard surface. Thus $\Sigma^{\prime}$ is isotopic in $M=X\left(s_{1}, \ldots, s_{k}\right)$ to the surface $\Sigma$ obtained by amalgamating the splitting $\left\{W_{1}, \ldots, W_{m}\right\}$. In other words, we have isotoped $\Sigma$ into $X\left(s_{1}, \ldots, s_{k-1}\right)$, in such a way that it is still a Heegaard surface. Repeating the above argument for the core $\gamma_{k-1} \subset X\left(s_{1}, \ldots, s_{k-1}\right)$, and so on, completes the proof of Theorem 1.1 .

\section{Acknowledgments}

We thank Marc Lackenby, Yo'av Rieck, Saul Schleimer, and Juan Souto for a number of helpful conversations. We also thank the referee for numerous suggestions that improved our exposition. Futer is supported in part by NSF grant DMS-1007221. Purcell is supported in part by NSF grant DMS-1007437 and the Alfred P. Sloan Foundation.

\section{References}

[1] I. Agol, Bounds on exceptional Dehn filling, Geom. Topol. 4 (2000), 431-449.

[2] S.A. Bleiler and C.D. Hodgson, Spherical space forms and Dehn filling, Topology 35(3) (1996), 809-833.

[3] W. Breslin, Short geodesics in hyperbolic 3-manifolds, Algebr. Geom. Topol. 11(2) (2011), 735-745.

[4] A.J. Casson and C.McA. Gordon, Reducing Heegaard splittings, Topol. Appl. 27(3) (1987), 275-283.

[5] J. Cerf, Sur les difféomorphismes de la sphère de dimension trois $\left(\Gamma_{4}=\right.$ 0), Lecture Notes in Mathematics, 53, Springer-Verlag, Berlin, 1968. 
[6] D. Cooper, D. Futer and J.S. Purcell, Dehn filling and the geometry of unknotting tunnels, Geom. Topol, to appear. arXiv:1105.3461.

[7] C. De Lellis and F. Pellandini, Genus bounds for minimal surfaces arising from min-max constructions, J. Reine Angew. Math. 644 (2010), 47-99.

[8] M. Freedman, J. Hass and P. Scott, Least area incompressible surfaces in 3-manifolds, Invent. Math. 71(3) (1983), 609-642.

[9] D. Futer, E. Kalfagianni and J.S. Purcell, Dehn filling, volume, and the Jones polynomial, J. Differ. Geom. 78(3) (2008), 429-464.

[10] W. Jaco and J. Hyam Rubinstein, Layered-triangulations of 3-manifolds, arXiv:math/0603601.

[11] M. Lackenby, Word hyperbolic Dehn surgery, Invent. Math. 140(2) (2000), 243-282.

[12] _ Heegaard splittings, the virtually Haken conjecture and property $(\tau)$, Invent. Math. 164(2) (2006), 317-359.

[13] _ An algorithm to determine the Heegaard genus of simple 3-manifolds with nonempty boundary, Algebr. Geom. Topol. 8(2) (2008), 911-934.

[14] J. Maher, Heegaard gradient and virtual fibers, Geom. Topol. 9 (2005), 2227-2259 (electronic).

[15] W. Meeks, III, L. Simon and S.T. Yau, Embedded minimal surfaces, exotic spheres, and manifolds with positive Ricci curvature, Ann. Math.

(2) 116(3) (1982), 621-659.

[16] Y. Moriah and H. Rubinstein, Heegaard structures of negatively curved 3-manifolds, Comm. Anal. Geom. 5(3) (1997), 375-412.

[17] J.T. Pitts and J.H. Rubinstein, Applications of minimax to minimal surfaces and the topology of 3-manifolds, Miniconference on geometry and partial differential equations, 2 (Canberra, 1986), Proc. Centre Math. Anal. Austral. Nat. Univ., vol. 12, Austral. Nat. Univ., Canberra, 1987, pp. 137-170.

[18] Y. Rieck, Heegaard structures of manifolds in the Dehn filling space, Topology 39(3) (2000), 619-641.

[19] Y. Rieck and E. Sedgwick, Finiteness results for Heegaard surfaces in surgered manifolds, Comm. Anal. Geom. 9(2) (2001), 351-367. 
[20] , Persistence of Heegaard structures under Dehn filling, Topol. Appl. 109(1) (2001), 41-53.

[21] J. Hyam Rubinstein, Minimal surfaces in geometric 3-manifolds, Global theory of minimal surfaces, Clay Math. Proc., vol. 2, Amer. Math. Soc., Providence, RI, 2005, pp. 725-746.

[22] J.H. Rubinstein and M. Scharlemann, Comparing Heegaard splittings of non-Haken 3-manifolds, Topology 35(4) (1996), 1005-1026.

[23] _ Comparing Heegaard splittings - the bounded case, Trans. Amer. Math. Soc. 350(2) (1998), 689-715.

[24] M. Scharlemann, Local detection of strongly irreducible Heegaard splittings, Topol. Appl. 90(1-3) (1998), 135-147.

[25] M. Scharlemann and A. Thompson, Thin position for 3-manifolds, Geometric topology (Haifa, 1992), Contemp. Math., vol. 164, Amer. Math. Soc., Providence, RI, 1994, pp. 231-238.

[26] R. Schoen and Shing Tung Yau, Existence of incompressible minimal surfaces and the topology of three-dimensional manifolds with nonnegative scalar curvature, Ann. Math. (2) 110(1) (1979), 127-142.

[27] J. Schultens, Heegaard splittings of Seifert fibered spaces with boundary, Trans. Amer. Math. Soc. 347(7) (1995), 2533-2552.

[28] J. Souto, Geometry, Heegaard splittings and rank of the fundamental group of hyperbolic 3-manifolds, Workshop on Heegaard Splittings, Geom. Topol. Monogr., vol. 12, Geom. Topol. Publ., Coventry, 2007, pp. 351-399. 
Department of Mathematics

TEMPle University

Philadelphia

PA 19122

USA

Department of Mathematics

Brigham Young UNIVERSITY

Provo

UT 84602

USA

E-mail address: dfuter@temple.edu

E-mail address: jpurcell@math.byu.edu

ReCeIVED ApRIL 26, 2012 